\title{
A Novel Method of Seizure Onset Zone Localization by Serial Tc-99m ECD Brain Perfusion SPECT Clearance Patterns
}

\author{
Chanan Sukprakun \\ King Chulalongkorn Memorial Hospital \\ Chusak Limotai \\ Chulalongkorn University Faculty of Medicine \\ Kitiwat Khamwan \\ Chulalongkorn University Faculty of Medicine \\ Panya Pasawang \\ King Chulalongkorn Memorial Hospital \\ Supatporn Tepmongkol ( $\sim$ supatporn@hotmail.com ) \\ Chulalongkorn University Faculty of Medicine https://orcid.org/0000-0002-6092-0488
}

\section{Research Article}

Keywords: Epilepsy, brain SPECT, ECD clearance, washout, ictal onset zone

Posted Date: July 7th, 2021

DOI: https://doi.org/10.21203/rs.3.rs-672209/v1

License: (c) (i) This work is licensed under a Creative Commons Attribution 4.0 International License. Read Full License 


\section{Abstract}

In this prospective study, we postulate that there is a difference between clearance of [99mTc]Tc- ethyl cysteinate dimer (ECD) in the seizure onset zone (SOZ) and other brain areas and thus SOZ localization by clearance patterns might become a potential novel method for SOZ localization in epilepsy. The parametric images of brain ECD clearance were generated by linear regression model analysis from serial brain SPECT scans from 30 minutes to 240 minutes after ECD injection (7-times point) in 7 patients with drug-resistant epilepsy and 3 normal volunteers. Clearance patterns of the SOZ confirmed by good surgical outcome or consensus with other investigations were analyzed quantitatively and semi-quantitatively by visual grading (slower or faster washout than contralateral brain regions). The average [ $\left.{ }^{99 \mathrm{~m}} \mathrm{Tc}\right] \mathrm{Tc}-$ ECD clearance rates of SOZs were $+1.08 \% \pm 2.57 \% / \mathrm{hr}$ (wash in), $-7.02 \% \pm 2.56 \% / \mathrm{hr}$ (washout), and $-5.37 \% \pm 1.71 \% / \mathrm{hr}$ (washout) in ictal, aura and interictal states, respectively. Paired t-tests between the $\mathrm{SOZ}$ and contralateral regions showed statistically significant difference ( $p=0.039$ in interictal state). Clearance patterns that can define the SOZs were 1$)$ wash in and slow washout on ictal slope, 2$)$ fast washout on aura slope and interictal slope with 100\% (6/6), 100\% (2/2) and 75\% (6/8) localization using ictal, aura, and interictal slope maps, respectively. Our study provided the evidence that clearance pattern methods are potential additive diagnostic tools for $\mathrm{SOZ}$ localization when routine one-time point SPECT are unable to define the SOZ.

\section{Introduction}

Almost 50 million patients were diagnosed with active epilepsy (Collaborators, 2019). Approximately 20-40\% of epilepsy becomes drugresistant epilepsy (DRE) (French, 2007). In one tertiary-care hospital in Thailand, the prevalence of DRE is 26.1\% (Towanabut et al., 1998). DRE or long-term epilepsy also increases mortality and decreases quality of life (Kanchanatawan \& Kasalak, 2012; Sillanpaa \& Shinnar, 2010).

Surgical treatment with correct localization of epileptogenic zone (EZ) in DRE shows better outcome than continued medical treatment (Ramey et al., 2013). There are generally 2 types of EZ localization, invasive and non-invasive methods. Intracranial electroencephalography (iEEG), an invasive method, is considered a gold standard for pre-surgical localization (Shah \& Mittal, 2014). Scalp EEG and single photon emission computed tomography (SPECT) are examples of non-invasive methods. In current practice, SPECT in epilepsy is performed at onetime point for ictal and interictal states. The sensitivity for localization by interictal SPECT alone is low (<50\%) in both temporal lobe epilepsy (TLE) and extra-temporal lobe epilepsy (ETE). The sensitivity of ictal SPECT is higher (70-90\% for TLE and 60-80\% for ETE) (Kumar \& Chugani, 2013; Weil et al., 2001). An additional technique that is usually used to improve seizure focus localization is called subtraction ictal SPECT coregistered to MRI (SISCOM). Even though SISCOM has high sensitivity (>80\%), sometimes the results are still inconclusive (Chen \& Guo, 2016; Kumar \& Chugani, 2013; Weil et al., 2001).

Technetium-99m ethyl cysteinate dimer ([99m Tc]Tc-ECD), a radiopharmaceutical used for brain SPECT imaging in epilepsy, is a lipophilic radiotracer. After injecting into the vein, it passively diffuses through blood-brain barrier (BBB), undergoes de-esterification, becomes hydrophilic and gets trapped in the brain cells. Its uptake not only associates with regional cerebral blood flow, but also reflects ictal neuronal synaptic activity (Leveille et al., 1989; Rabinowicz et al., 1997). [99mTc]Tc-ECD reaches maximum activity in the brain within a few minutes and remains near that level for the next 10-20 mins evidenced by both planar and SPECT imaging (Ishizu et al., 1996; Rabinowicz et al., 1997; Vallabhajosula et al., 1989). The average ECD washout or clearance rate was about - 6\%/hr during the first 6 hours in normal subjects (Leveille et al., 1989). The factor that affects clearance or washout of [ $\left.{ }^{99 \mathrm{~m}} \mathrm{Tc}\right] \mathrm{Tc}-\mathrm{ECD}$ is not well understood. There is one in vitro study supporting the hypothesis that esterase activity is the major determinant of the overall retention of ECD (Jacquier-Sarlin, Polla, \& Slosman, 1996). Evidence about regional variation for clearance of [99m Tc]Tc-ECD in normal brain are controversial (Flores et al., 1999; Ichise et al., 1997; Leveille et al., 1989). In a study of epilepsy, [ ${ }^{99 m}$ Tc]Tc-ECD washout rate was reported to be slightly lower but statistically significant in the hyperperfused areas than in other brain areas. However, this washout rate was measured from only two static ictal SPECT at 30 minutes and the next 40 minutes. In this study, only five patients underwent ictal SPECT twice (Grunwald et al., 1994). Thus, clearance rate from each brain region, especially the seizure onset zone (SOZ), still needs evaluation.

In this study, we postulate that there is a difference between clearance of [ $\left.{ }^{99 m} \mathrm{Tc}\right] \mathrm{Tc}-\mathrm{ECD}$ in the SOZ and other brain areas and thus seizure focus localization by clearance patterns might become a potential non-invasive method for better pre-surgical SOZ localization.

\section{Material And Methods Subjects}


All DRE patients aged 18-60 years who were sent for ictal and interictal SPECT scans from Mar 2018 to Sep 2019 and normal volunteers without history of neurologic or psychiatric disorder were enrolled and informed consent was obtained. We excluded patients with history or MRI of cerebrovascular disease or psychiatric disorder. This study received ethics committee approval by The Institutional Review Board of the Faculty of Medicine, Chulalongkorn University, Bangkok, Thailand (COA No. 078/2018 and IRB No. 680/60).

\section{Image acquisition}

[ ${ }^{99} \mathrm{~m}$ Tc]Tc-ECD (Neurolite) with radioactivity of $0.3 \mathrm{mCi} / \mathrm{kg}$ diluted in $10 \mathrm{ml}$ of NSS was injected intravenously using bolus technique followed by $10 \mathrm{ml}$ of NSS flush. For DRE patients, [ $\left.{ }^{99 \mathrm{~m}} \mathrm{Tc}\right] \mathrm{Tc}-\mathrm{ECD}$ was injected under EEG monitoring in both ictal (within 60 seconds after EEG or clinical onset) and interictal (more than 24 hours after last seizure) states to confirm patient's seizure state. Aura state was defined as a subjective ictal phenomenon without definite ictal EEG onset.

Serial brain SPECT scanning was performed on a dual-head gamma camera (Siemens/Evo Excel, Erlangen, Germany) with high-resolution, fan-beam collimators and $140 \mathrm{keV} \pm 15 \%$ energy window by static scan mode at 3-degree angle interval (180 degrees for each head $=60$ projections, 20 seconds per projection, 20 minutes per scan) at 30, 90, 120, 150, 180, 210 and 240-minute postinjection. All images were reconstructed by a filtered back-projection (FBP) algorithm with a Butterworth filter (cutoff frequency 0.35 , power factor 5 ) and displayed on a 128 x 128 matrix. The pixel size was $3.3 \times 3.3 \mathrm{~mm}$, and the slice thickness was $3.3 \mathrm{~mm}$. Attenuation correction was performed by Chang's method with an attenuation coefficient equal to $0.12 \mathrm{~cm}^{-1}$.

\section{Data analysis}

\section{Image analysis}

All reconstructed images were decay corrected by Tc-99m physical half-life (6.03hr), and then merged into time-sequence images. These merged-images were motion corrected (between-runs movements) and further co-registered to patient's T1-weighted MRI images for anatomical correlation. These images then underwent a pixelwise linear regression analysis. The model yielded a slope map, which is a picture of the brain that reflects the clearance rate from each image pixel. The grey matter (GM) image was created from the patient's T1weighted MRI to further mask the slope map. Finally, the regions of interest (ROIs) of each brain region (except the SOZ) were defined by the automated anatomical labeling (AAL) template atlas (Tzourio-Mazoyer et al., 2002). ROls of the SOZs were drawn by iso-contour (3D isocontouring VOI tools using region-growing) inside AAL template over the suspected regions defined by the Engel class I surgical outcome (free of disabling seizures) (Engel, 1993) for those who had surgery, or consensus from epilepsy conference using iEEG, scalp EEG and/or MRI lesions in patients without surgery. Mirroring of ROIs of the SOZs to the contralateral regions of the SOZs was done for comparison. All ROIs were applied by the same investigator (C.S.) to eliminate the interoperator variation. These ROls were verified by second investigator (S.T.) who was blinded to clinical data and other imaging results. All image analyses were done using PMOD software (version 4.101, PMOD Technologies Ltd., Zürich, Switzerland) as illustrated in Fig. 1.

\section{Image interpretation}

The patterns of slope maps were visually interpreted and classified by semi-quantitative method into four categories; 1 . wash in (positive rate), 2. slow washout (negative rate with clearance rate slower than contralateral region), 3. normal washout (negative rate with clearance rate equal to contralateral region), and 4 . fast washout (negative rate with clearance rate faster than contralateral region). Positive results were considered when there were patterns other than normal washout pattern. Only normal washout pattern, which was the washout pattern equal to contralateral mirrored ROI region, was considered a negative result. The slope map was displayed in split BWR (blue-white-red) color bar. Example of slow washout pattern is illustrated in Fig. 2.

\section{Statistical analysis}

Paired t-test was used to compare ECD clearance rates of the SOZ and contralateral regions in each patient seizure state, i.e. ictal and interictal states, and to compare ECD clearance rates of the SOZs between ictal and interictal scans. F-test, repeated-measures analysis of variance (ANOVA) was used in 3 analyses: 1) to compare each brain regions among normal brains, 2) to compare whole brain clearance among each seizure state and normal brains, 3) to compare ECD clearance rates of SOZ and contralateral homologous brain region among each state of seizure in patients and mean value of normal brains in the same region as SOZ Statistical significance was defined as $\mathrm{p}<$ 0.05 for all analyses. All statistical analyses were performed by SPSS version 22 software for Microsoft Windows.

\section{Results}




\section{Enrollment}

A total of 12 cases, 9 patients with DRE and 3 normal volunteers were enrolled. Two patients were excluded due to history of CVA and severe head trauma with craniotomy. There were 17 serial brain SPECT scans in 10 subjects; 5 were performed in ictal state, 2 were performed in aura state, 7 were performed in interictal state, and 3 were performed in 3 normal volunteers in resting state.

In seven patients, the median age was 28 (range 20-47) years. The male/female ratio was 3:4. All patients had been diagnosed as DRE and suffered from persistent seizure for more than five years (median 16, range 6-27 years). The TLE/ETE ratio was 4:3. Five of seven patients (71.4\%) underwent surgical treatment and later had Engel class I at least 15-month follow-up except one patient underwent partial lesionectomy due to neurological deficit concern. Patient characteristics and demographic data are summarized in Table 1. 
Table 1

Patient characteristics and Demographic data

\begin{tabular}{|c|c|c|c|c|c|c|c|}
\hline No. & $\begin{array}{l}\text { Age/Sex } \\
\text { (onset } \\
\text { age) }\end{array}$ & Seizure type & $\begin{array}{l}\text { Seizure } \\
\text { frequency } \\
\text { (times/duration) }\end{array}$ & Medication & $\begin{array}{l}\text { Provisional } \\
\text { diagnosis }\end{array}$ & $\begin{array}{l}\text { Gold } \\
\text { standard }\end{array}$ & $\begin{array}{l}\text { Surgical } \\
\text { procedure } \\
\text { (pathology) }\end{array}$ \\
\hline 1 & $\begin{array}{l}26 / \mathrm{M} \\
(20)\end{array}$ & $\begin{array}{l}\text { - GTC } \\
\text { - FSIC with automatism } \\
\text { - Aura (left-sided auditory } \\
\text { and somatosensory) } \diamond \pm \\
\text { FMS (left face) } \\
\text { - Mnemonic aura (jamais } \\
\text { vu) }\end{array}$ & $\begin{array}{l}\sim 1-2 \text { /year } \\
\text { - rare } \\
\sim 3-10 \text { /month } \\
\sim 1-2 / \text { month }\end{array}$ & $\begin{array}{l}\text { CBZ, CLB, LVT, } \\
\text { PRP, TPM, } \\
\text { pyridoxine }\end{array}$ & TLE & $\begin{array}{l}\text { Surgical } \\
\text { outcome } \\
\text { (Engel } \\
\text { II) }\end{array}$ & $\begin{array}{l}\text { Right superior } \\
\text { and middle } \\
\text { temporal gyri } \\
\text { partial } \\
\text { lesionectomy } \\
\text { (mild MCD, } \\
\text { oligodendroglial } \\
\text { hyperplasia) }\end{array}$ \\
\hline 2 & $\begin{array}{l}47 / F \\
(20)\end{array}$ & $\begin{array}{l}\text { - Aura (autonomic c/s } \\
\text { experiential) with FSIC } \\
\text { and vocalization }\end{array}$ & $\sim 1-2$ /month & $\begin{array}{l}\text { CLB, CBZ, LVT, } \\
\text { PHT, TPM }\end{array}$ & TLE & $\begin{array}{l}\text { scalp } \\
\text { EEG + } \\
\text { MRI } \\
\text { lesion }\end{array}$ & $\mathrm{n} / \mathrm{a}$ \\
\hline 3 & $\begin{array}{l}31 / F \\
(10)\end{array}$ & $\begin{array}{l}\text { - Aura with FSIC with } \\
\text { oral automatism } \\
\text { - Aura alone (dizziness } \pm \\
\text { fear) }\end{array}$ & $\begin{array}{l}\sim 4 \text { /month } \\
\sim 2 \text { /month }\end{array}$ & $\begin{array}{l}\text { CBZ, LVT, PB, PHT, } \\
\text { TPM }\end{array}$ & TLE & $\begin{array}{l}\text { Surgical } \\
\text { outcome } \\
\text { (Engel I) }\end{array}$ & $\begin{array}{l}\text { Right AMTL } \\
\text { (hippocampal } \\
\text { sclerosis, } \\
\text { ILAE type I, mild } \\
\text { oligodendroglial } \\
\text { hyperplasia) }\end{array}$ \\
\hline 4 & $\begin{array}{l}42 / F \\
(16)\end{array}$ & $\begin{array}{l}\text { - Aura with dyscognitive } \\
\text { (impaired awareness) } \\
\text { with automatism } \\
\text { - Aura alone (dejavu) }\end{array}$ & $\begin{array}{l}\sim 5-6 / \text { month } \\
\sim 2-3 \text { /month }\end{array}$ & LMT, LVT & TLE & $\begin{array}{l}\text { scalp } \\
\text { EEG }\end{array}$ & $\mathrm{n} / \mathrm{a}$ \\
\hline 5 & $\begin{array}{l}20 / F \\
(13)\end{array}$ & $\begin{array}{l}\text { - Nocturnal GTC } \\
\text { - Aura (chest tightness and } \\
\text { left-sided face numbness) } \\
\text { with bilateral arm tonic }\end{array}$ & $\begin{array}{l}\sim 3-4 / \text { month } \\
\sim 10-20 \text { /day }\end{array}$ & $\begin{array}{l}\text { CBZ, LCS, LVT, PB, } \\
\text { TPM, VPA, } \\
\text { prednisolone, } \\
\text { azathioprine }\end{array}$ & ETE & $\begin{array}{l}\text { Surgical } \\
\text { outcome } \\
\text { (Engel I) }\end{array}$ & $\begin{array}{l}\text { Right middle } \\
\text { frontal gyrus } \\
\text { lesionectomy } \\
\text { (FCD type lla) }\end{array}$ \\
\hline 6 & $\begin{array}{l}25 / \mathrm{M} \\
(14)\end{array}$ & $\begin{array}{l} \pm \text { Aura with FSIC with both } \\
\text { hand tonic } \pm \text { vocalization } \\
\text { - Dialeptic }\end{array}$ & $\begin{array}{l}\sim 15 \text { /month } \\
\sim 1 / 2-3 \text { month }\end{array}$ & $\begin{array}{l}\text { LVT, CBZ, LCS, } \\
\text { CNZ }\end{array}$ & ETE & $\begin{array}{l}\text { Surgical } \\
\text { outcome } \\
\text { (Engel I) }\end{array}$ & $\begin{array}{l}\text { Left posterior } \\
\text { cingulate gyrus } \\
\text { (low grade } \\
\text { diffuse } \\
\text { astrocytoma } \\
\text { WHO grade II) }\end{array}$ \\
\hline 7 & $\begin{array}{l}28 / \mathrm{M} \\
(12)\end{array}$ & $\begin{array}{l}\text { - Nocturnal GTC } \\
\text { - Aura (dizziness, blurred } \\
\text { vision) with FSIC and } \\
\text { automatism }\end{array}$ & $\begin{array}{l}\sim 1 / \text { month } \\
\sim 1-2 / \text { month }\end{array}$ & $\begin{array}{l}\text { CBZ, CLB, LVT, PB, } \\
\text { PHT }\end{array}$ & ETE & $\begin{array}{l}\text { Surgical } \\
\text { outcome } \\
\text { (Engel I) }\end{array}$ & $\begin{array}{l}\text { Left fusiform } \\
\text { gyrus } \\
\text { lesionectomy } \\
\text { (low grade } \\
\text { diffuse } \\
\text { astrocytoma } \\
\text { WHO grade II) }\end{array}$ \\
\hline \multicolumn{8}{|c|}{ a This patient underwent partial lesionectomy due to neurological deficit concern. } \\
\hline $\begin{array}{l}\text { GTC } \\
\text { carb } \\
\text { pher } \\
\text { elec } \\
\text { lobe }\end{array}$ & $\begin{array}{l}\text { generalizec } \\
\text { mazepine, } \\
\text { toin, PRP: } \\
\text { oencephalc } \\
\text { tomy, ILAE: }\end{array}$ & $\begin{array}{l}\text { tonic-clonic seizure, FSIC: foc } \\
\text { eB: clobazem, CNZ: clonazep } \\
\text { erampanel, TPM: topiramate, } \\
\text { graphy, MRI: magnetic resona } \\
\text { nternational league against e }\end{array}$ & $\begin{array}{l}\text { I seizures with imp } \\
\text { m, LCS: lacosamid } \\
\text { PA: valproic acid, } \\
\text { ce imaging, MCD: r } \\
\text { lepsy, FCD: focal c }\end{array}$ & $\begin{array}{l}\text { ired consciousness, } \\
\text { L LMT: lamotrigine, L } \\
\text { LE: temporal lobe ep } \\
\text { alformation of corti } \\
\text { rtical dysplasia, WH }\end{array}$ & $\begin{array}{l}\text { IS: focal mot } \\
\text { r: levotiracet } \\
\text { psy, ETE: ext } \\
\text { developmer } \\
\text { world health }\end{array}$ & $\begin{array}{l}\text { seizures, C } \\
\text { n, PB: phen } \\
\text {-temporal Ic } \\
\text { AMTL: ante } \\
\text { rganization }\end{array}$ & $\begin{array}{l}\text { Z: } \\
\text { barbital, PHT: } \\
\text { oe epilepsy, EEG: } \\
\text { omesial temporal }\end{array}$ \\
\hline
\end{tabular}

In three normal volunteers, the median age was 30 (range 29-43) years. The male/female ratio was 1:2.

\section{SPECT image acquisition}


In a total of 119 static SPECT scans (17 serial SPECT scans $x$ 7-time static each), 6 (5.3\%) were excluded due to delayed patient transfer to Nuclear Medicine. Of the remaining 113 static scans, 10 (8 scans from patients' images and 2 scans from normals' images, 8.8\%) were further excluded from analysis due to within-runs movements detected after image reconstruction (Supplementary Table A1). The delay time between seizure onset and $\left[{ }^{99 \mathrm{~m}} \mathrm{Tc}\right] \mathrm{Tc}-\mathrm{ECD}$ injection was 4-38 seconds with average injected dose of $641.7 \pm 127.0 \mathrm{MBq}(17.3 \pm 3.4$ $\mathrm{mCi})$. There was no detectable clinical seizure during the serial scan.

\section{Clearance rates and patterns}

\section{Normal group}

The average $\left[{ }^{99 m} \mathrm{Tc}\right] \mathrm{Tc}$-ECD clearance rate from whole brain was $-3.15 \% \pm 0.55 \% / \mathrm{hr}$ (mean \pm S.D.). Repeated-measures ANOVA analysis showed no statistically significant difference in clearance rates among each brain region (Table 2).

Table 2

ECD clearance rate $(\% / h r)$ in three normal brains

\begin{tabular}{|lllllllllll|}
\hline \multicolumn{10}{|c|}{ Region } \\
\hline & Age & Sex & Frontal & Temporal & Parietal & Occipital & Cerebellum & Basal ganglia & Thalamus & $\begin{array}{l}\text { Whole } \\
\text { brain (GM) }\end{array}$ \\
\hline N1 & 30 & F & -3.67 & -4.00 & -3.71 & -3.46 & -3.79 & -3.46 & -2.60 & -3.71 \\
\hline N2 & 29 & M & -2.82 & -2.58 & -2.25 & -2.59 & -2.80 & -2.18 & -1.40 & -2.61 \\
\hline N3 & 43 & F & -3.42 & -3.22 & -2.87 & -2.82 & -3.19 & -3.18 & -2.20 & -3.14 \\
\hline Mean & 34.0 & M:F & -3.30 & -3.27 & -2.95 & -2.95 & -3.26 & -2.94 & -2.06 & -3.15 \\
\hline S.D. & 7.8 & $1: 2$ & 0.44 & 0.71 & 0.73 & 0.45 & 0.50 & 0.67 & 0.61 & 0.55 \\
\hline
\end{tabular}

\section{Patient group}

The average [99m Tc]Tc-ECD clearance rate from whole brain were $-2.52 \% \pm 0.68 \% / \mathrm{hr},-3.29 \% \pm 0.18 \% / \mathrm{hr}$, and $-3.74 \% \pm 1.07 \% / \mathrm{hr}(\mathrm{mean}$ \pm S.D.) in ictal, aura and interictal states, respectively. Repeated-measures ANOVA analysis showed no statistically significant difference in whole brain clearance rates among these three states and normal volunteers.

There were $8 \mathrm{SOZs}$ in seven patients (One patient had bilateral temporal seizure onsets.). The average $\left[{ }^{99 \mathrm{~m}} \mathrm{Tc}\right] \mathrm{Tc}-\mathrm{ECD}$ clearance rate of all SOZs were $+1.08 \% \pm 2.57 \% / \mathrm{hr}$ (wash in), $-7.02 \% \pm 2.56 \% / \mathrm{hr}$, and $-5.37 \% \pm 1.71 \% / \mathrm{hr}$ in ictal, aura and interictal states, respectively.

Repeated-measures ANOVA analysis showed a statistically significant difference among SOZ clearance rate of three seizure states in patients and normal volunteers $(p<0.001)$. Subgroup analysis showed a statistically significant difference between ictal and aura, ictal and interictal, and ictal and normal. There was no statistically significant difference among aura, interictal and normal pairs. Values of the SOZ clearance rates and patterns in each patient are summarized in Table 3. Examples of patient slope maps are illustrated in Fig. 3. 
Table 3

Clearance patterns and clearance rates in seizure onset zone (SOZ) defined by gold standard.

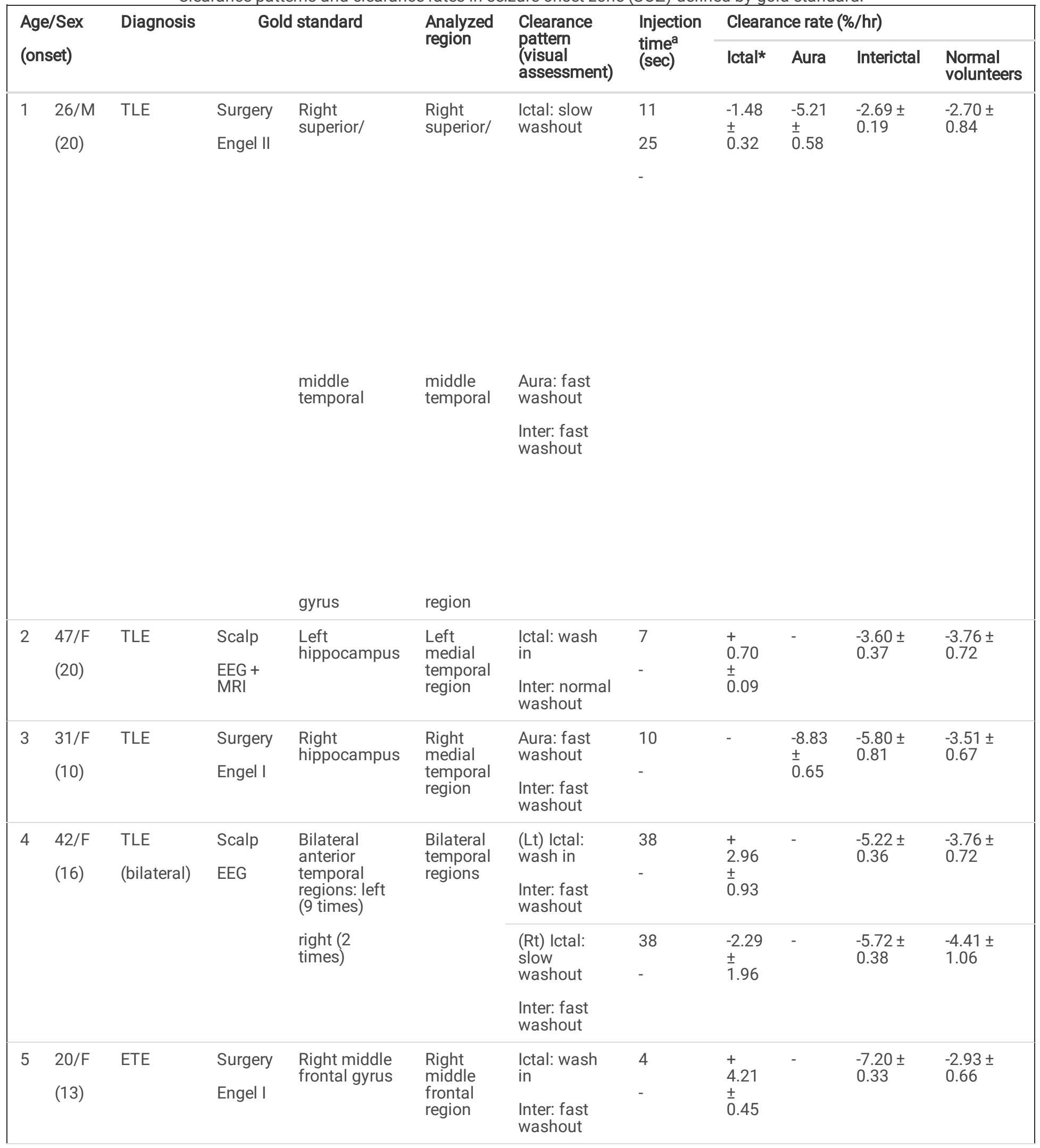

a Injection time of ECD after EEG or clinical onset for ictal and aura states

* Ictal state showed statistically significant difference $(p<0.001)$ from aura, interictal, and normal. No statistically significant difference among aura, interical and normal

TLE: temporal lobe epilepsy, ETE: extra-temporal lobe epilepsy, EEG: electroencephalography, MRI: magnetic resonance imaging 


\begin{tabular}{|c|c|c|c|c|c|c|c|c|c|c|c|}
\hline \multirow{2}{*}{\multicolumn{2}{|c|}{$\begin{array}{l}\text { Age/Sex } \\
\text { (onset) }\end{array}$}} & \multirow{3}{*}{$\begin{array}{l}\text { Diagnosis } \\
\\
\text { ETE }\end{array}$} & \multirow{2}{*}{\multicolumn{2}{|c|}{ Gold standard }} & \multirow{3}{*}{$\begin{array}{l}\begin{array}{l}\text { Analyzed } \\
\text { region }\end{array} \\
\text { left } \\
\text { posterior } \\
\text { cingulate } \\
\text { region }\end{array}$} & \multirow{3}{*}{$\begin{array}{l}\begin{array}{l}\text { Clearance } \\
\text { pattern } \\
\text { (visual } \\
\text { assessment) }\end{array} \\
\text { Ictal: wash } \\
\text { in } \\
\text { Inter: normal } \\
\text { washout }\end{array}$} & \multirow{3}{*}{$\begin{array}{l}\text { Injection } \\
\text { time } \\
\text { (sec) } \\
16\end{array}$} & \multicolumn{4}{|c|}{ Clearance rate $(\% / h r)$} \\
\hline & & & & & & & & \multirow{2}{*}{$\begin{array}{l}\text { Icta|* } \\
+ \\
+2.37 \\
\pm \\
0.10\end{array}$} & \multirow{2}{*}{$\begin{array}{l}\text { Aura } \\
-\end{array}$} & \multirow{2}{*}{$\begin{array}{l}\text { Interictal } \\
-4.86 \pm \\
0.41\end{array}$} & \multirow{2}{*}{$\begin{array}{l}\begin{array}{l}\text { Normal } \\
\text { volunteers }\end{array} \\
-3.38 \pm \\
0.39\end{array}$} \\
\hline 6 & $25 / M$ & & Surgery & $\begin{array}{l}\text { Left posterior } \\
\text { cingulate } \\
\text { gyrus }\end{array}$ & & & & & & & \\
\hline & $(14)$ & & Engel I & & & & - & & & & \\
\hline 7 & $\begin{array}{l}28 / \mathrm{M} \\
(12)\end{array}$ & ETE & $\begin{array}{l}\text { Surgery } \\
\text { Engel I }\end{array}$ & $\begin{array}{l}\text { Left fusiform } \\
\text { gyrus }\end{array}$ & $\begin{array}{l}\text { Left } \\
\text { fusiform } \\
\text { region }\end{array}$ & $\begin{array}{l}\text { Inter: fast } \\
\text { washout }\end{array}$ & - & - & - & $\begin{array}{l}-7.85 \pm \\
1.17\end{array}$ & $\begin{array}{l}-3.73 \pm \\
0.32\end{array}$ \\
\hline \multicolumn{12}{|c|}{ a Injection time of ECD after EEG or clinical onset for ictal and aura states } \\
\hline \multicolumn{12}{|c|}{$\begin{array}{l}\text { *Ictal state showed statistically significant difference }(p<0.001) \text { from aura, interictal, and normal. No statistically significant difference } \\
\text { among aura, interical and normal }\end{array}$} \\
\hline
\end{tabular}

The average [ $\left.{ }^{99 \mathrm{~m}} \mathrm{Tc}\right] \mathrm{Tc}-\mathrm{ECD}$ clearance rate of the regions contralateral to all SOZs were $-2.75 \% \pm 1.73 \% / \mathrm{hr},-1.51 \% \pm 0.74 \% / \mathrm{hr}$, and -3.21 $\% \pm 1.25 \% / \mathrm{hr}$ in ictal, aura and interictal states, respectively. Paired t-tests between clearance rate of the SOZ and contralateral regions showed statistically significant difference in interictal state $(p=0.039)$, but no statistically significant difference were seen in ictal state, aura state and normal. Comparison of clearance rates of the individual values of the clearance rate of the SOZs and the contralateral regions in three seizure states and average clearance rate of the same regions in normal volunteers are demonstrate in Fig. 4. Detailed values can be seen in the Supplementary Table A2.

\section{Percent localization of epileptogenic focus}

From ictal slope maps, there were two slope patterns in $6 \mathrm{SOZs}$, which were 4 washin and 2 slow washout patterns. From aura slope maps, there was only one slope pattern in $2 \mathrm{SOZs}$, which was fast washout pattern. From interictal slope maps, there were two slope patterns in 8 SOZs, including 2 normal washout and 6 fast washout patterns. Thus, 6 of 8 lesions were considered interictal positive slope map pattern. Therefore, these maps resulted in $100 \%(6 / 6), 100 \%(2 / 2)$, and $75 \%(6 / 8)$ localization for ictal, aura, and interictal, respectively. The percent localizations were $62.5 \%$ (5/8) by MRI, $37.5 \%$ (3/8) by visual SPECT, 33.3\% (2/6) by SISCOM and 37.5\% (3/8) by visual SPECT with SISCOM. Comparison of percent localization among imaging methods, including MRI, visual SPECT, SISCOM, visual SPECT plus SISCOM, ictal, aura, interictal slope maps and combination of slope maps in all three states were summarized in Table 4. 
Table 4

Comparison of imaging methods and percent localization of the seizure onset zones

\begin{tabular}{|c|c|c|c|c|c|c|c|c|c|c|}
\hline & $\begin{array}{l}\text { Epileptogenic or } \\
\text { Seizure onset } \\
\text { zone }\end{array}$ & Gold standard & MRI & $\begin{array}{l}\text { Visual } \\
\text { SPECT }\end{array}$ & SISCOM & $\begin{array}{l}\text { Visual } \\
\text { SPECT + } \\
\text { SISCOM }\end{array}$ & $\begin{array}{l}\text { Interictal } \\
\text { slope }\end{array}$ & $\begin{array}{l}\text { Aura } \\
\text { slope }\end{array}$ & $\begin{array}{l}\text { Ictal } \\
\text { slope }\end{array}$ & $\begin{array}{l}\text { Combination } \\
\text { of slope }\end{array}$ \\
\hline P1 & $\begin{array}{l}\text { Right superior/ } \\
\text { middle temporal } \\
\text { gyrus }\end{array}$ & surgery & 0 & 0 & 0 & 0 & $\Pi$ & $\Pi$ & $\Pi$ & $\Pi$ \\
\hline P2 & $\begin{array}{l}\text { Left } \\
\text { hippocampus }\end{array}$ & scalp EEG + MRI & $\Pi$ & $\Pi$ & $\Pi$ & $\Pi$ & 0 & - & $\Pi$ & $\Pi$ \\
\hline P3 & $\begin{array}{l}\text { Right } \\
\text { hippocampus }\end{array}$ & surgery & $\Pi$ & 0 & - & 0 & $\Pi$ & $\Pi$ & - & $\Pi$ \\
\hline P4 & $\begin{array}{l}\text { Left temporal } \\
\text { region }\end{array}$ & scalp EEG & 0 & 0 & 0 & 0 & $\Pi$ & - & $\Pi$ & $\Pi$ \\
\hline P4 & $\begin{array}{l}\text { Right temporal } \\
\text { region }\end{array}$ & scalp EEG & 0 & $\Pi$ & $\Pi$ & $\Pi$ & $\Pi$ & - & $\Pi$ & $\Pi$ \\
\hline P5 & $\begin{array}{l}\text { Right middle } \\
\text { frontal gyrus }\end{array}$ & surgery & $\Pi$ & 0 & 0 & 0 & $\Pi$ & - & $\Pi$ & $\Pi$ \\
\hline P6 & $\begin{array}{l}\text { Left posterior } \\
\text { cingulate gyrus }\end{array}$ & Surgery & $\Pi$ & $\Pi$ & 0 & $\Pi$ & 0 & - & $\Pi$ & $\Pi$ \\
\hline P7 & $\begin{array}{l}\text { Left fusiform } \\
\text { gyrus }\end{array}$ & Surgery & $\Pi$ & 0 & - & 0 & $\Pi$ & - & - & $\Pi$ \\
\hline \multicolumn{3}{|c|}{ congruent results (n) } & $5 / 8$ & $3 / 8$ & $2 / 6$ & $3 / 8$ & $6 / 8$ & $2 / 2$ & $6 / 6$ & $8 / 8$ \\
\hline \multicolumn{3}{|c|}{$\begin{array}{l}\% \text { correct } \\
\text { localization }\end{array}$} & 62.5 & 37.5 & 33.33 & 37.5 & 75 & 100 & 100 & 100 \\
\hline$\Pi$ & $\begin{array}{l}=\text { congruent } \\
\text { standard }\end{array}$ & sult to gold & & $\begin{array}{l}\text { Icongruer } \\
\text { ndard }\end{array}$ & result to $\mathrm{g}$ & & - & $=\operatorname{not} p$ & formed & \\
\hline
\end{tabular}

\section{Discussion}

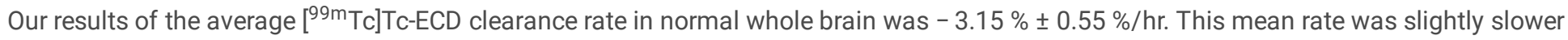
than in Léveillé, et al ( -6\%/hr) and Ischise, et al $(-4.3 \% \pm 1.7 \% / \mathrm{hr})$ studies (Leveille et al., 1989; Ichise et al., 1997). The thalamic clearance rate was slower than other brain regions in all three subjects, which is similar to the study by Ischise, et al. However, there was no statistically significant difference among regions in our study. The occipital clearance rate was similar to the rest of the brain regions, unlike Ischise's study whose findings showed significantly faster washout at this region. A possible explanation is the difference in methodological techniques such as image reconstruction and/or image analysis (curve fitting technique: monoexponential vs linear regression, or different ROIs templates: AAL atlas vs ellipses). Moreover, our rates were calculated from only three normal subjects, in contrast to twenty subjects used in their study.

The average whole brain ECD clearance rates of patients with DRE were -2.52 to $-3.74 \%$ in ictal, aura and interictal states, which were much slower than the clearance rate of DRE patients in Grunwald, et al's study (-8.9 to -13.5\%) (Grunwald et al., 1994). There are 2 possible explanations for this matter. Firstly, there were some differences in the aspects of acquisition time, namely number of serial SPECT scans (2-time vs 7-time point) and duration of scan (about 70 vs 240 minutes). Secondly, different ROI techniques were used. Grunwald's study might include soft tissue or background activities in their ROI, that may cause fast washout in their study. Therefore, there might be actual difference between early and late clearance rates or the clearance rate difference may be the result of interfered rapid soft tissue and background activities washout.

In contrast to other studies, our study further focused on the SOZ. We analyzed the SOZ using 2 methods: 1) comparison of epilepsy states in the same SOZ, and 2) comparison of SOZ with contralateral region in ictal, aura, and interictal states. In the former method, the average ECD clearance rate of the SOZs in ictal state showed a statistically significant difference $(p<0.001)$ from aura, interictal, and normal, while there was no statistically significant difference among aura, interical and normal (Table 3). This information shows that by using the first method, ictal state is crucial for comparison with other states to identify SOZ. The area with different clearance pattern from either interictal or aura state or normal brain is likely to be a SOZ. In the latter method, the SOZs showed statistically significant differences from the 
contralateral regions with $p=0.039$ in interictal state (Fig. 4). No statistically significant difference in aura state was detected. This is likely resulted from a small sample size $(\mathrm{N}=2)$ in aura state. Since the clearance pattern of aura is similar to interictal state, adding aura to interictal state would result in statistically significant difference from the contralateral regions with $p=0.011$. Thus, by using the latter method, either interictal or aura SPECT study may be used independently from ictal SPECT study when there is unavailable ictal SPECT study.

By visual analysis, clearance patterns those had localization performance were washin and slow washout patterns in ictal slope maps, fast washout patterns in aura slope maps and interictal slope maps. Washout rate was mentioned "slower" in the ictal onset zone (hyperperfused areas) than other brain areas in Grünwald F, et al. study. Surprisingly, our study also found washin patterns other than slow washout. Some probable explanations were that there might be some alteration in BBB permeability, regional cerebral blood flow and/or esterase activity causing more retention of [ ${ }^{99 m}$ Tc]Tc-ECD in the brain cells during ictal period and hours thereafter. Fast washout pattern was observed only in aura and interictal slope map. Mechanism of fast washout was probably due to low esterase activity in the SOZs, resulting in reduced ECD retention which was previously explained in vitro by Jacquier-Sarlin, et al's study (Jacquier-Sarlin et al., 1996). Aura also showed fast washout, which is similar to interictal slope map pattern. This may be explained by the state of aura itself, which is a subjective ictal phenomenon without definite ictal EEG onset, and thus resulting in patterns mimicking interictal state.

From our hospital statistics in the last 5 years, $40 \%$ of patients with DRE failed to complete both ictal and interical SPECT scans while they were hospitalized for video-EEG monitoring, resulting in only available interictal SPECT study which is almost useless for SOZ localization by conventional SPECT method. In this study, two of seven patients could not perform ictal SPECT because of rare seizure frequency. We have shown that multiple time points interictal SPECT scan is also useful and can improve SOZ localization as compared to only one-time point conventional interictal scan. Thus, having only interictal scan, SOZ can still be diagnosed if fast washout pattern is seen on serial SPECT interictal scan (Table 3, Fig. 3).

From our data, SOZ localization using slope maps showed that this method had better performance when compared to conventional imaging methods (Table 4). Thus, it is a promising method to increase percent localization of SOZ and should be performed in patients with discordant results of conventional pre-surgical investigations.

\section{Limitation and recommendation}

Correlation between injection time and clearance patterns or localization performance, which is one factor that affected biodistribution of ECD, could not be done due to the small sample size. For technical aspects, there are some drawbacks of this method: 1) Patients need to stay still during each static scan in order to complete serial SPECT scan. 2) Scan time was increased up to 4 hours from the routine 30-60 minutes, which may cause machine availability problems in the center with limited number of SPECT machines. Future study using 2 time points for clearance pattern analysis may be more practical in routine clinical practice. Analysis of clearance pattern in different pathology may provide better understanding of biodistribution of radiotracer in patient with DRE.

\section{Conclusion}

Clearance patterns that can define the SOZs are 1) wash in and slow washout in ictal state 2) fast washout in aura state and interictal state. This novel clearance pattern method showed additional benefit to the routine brain perfusion SPECT diagnosis for better SOZ localization.

\section{Declarations}

\section{Acknowledgments}

The authors would like to express their gratitude to Prof. Patrick Dupont and Dr. Geoff Warnock for data analysis counseling, to Dr. Suda Jirasakuldej and EMU team for taking care of the patients and sending patients for recruitment, to Research Affairs Department, Faculty of Medicine, Chulalongkorn University for providing statistical consultation and English editing, to Assoc.Prof.Tawachai Chaiwatanarat for his useful comments regarding the proposal, and to all Nuclear Medicine technologists in King Chulalongkorn Memorial Hospital for their help in data collection and image acquisition.

\section{Funding}

This work was supported by Ratchadapiseksompotch Fund, Faculty of Medicine, Chulalongkorn University, Bangkok, Thailand [grant number RA61/035]. 


\section{Conflicts of interest/Competing interests}

None of the authors have a conflict of interest to declare.

\section{Ethics approval}

This study was approved by the Institutional Review Board of the Faculty of Medicine, Chulalongkorn University, Bangkok, Thailand (COA No. $078 / 2018$ and IRB No. $680 / 60$ )

\section{Consent to participate}

Informed consent was obtained from all individual participants included in the study.

\section{Consent for publication}

Informed consent was obtained from all individual participants included in the study.

\section{Availability of data and material}

Data are available on request to the authors.

\section{Code availability}

Not applicable

\section{Author Contributions}

Author contributions included conception and study design (CS and ST), data collection or acquisition (CS, CL, KK and PP), statistical analysis (CS), interpretation of results (CS, KK and ST), drafting the manuscript work or revising it critically for important intellectual content (CS and ST) and approval of final version to be published and agreement to be accountable for the integrity and accuracy of all aspects of the work (All authors).

\section{References}

1. Collaborators, G. B. D. E. (2019). Global, regional, and national burden of epilepsy, 1990-2016: a systematic analysis for the Global Burden of Disease Study 2016. Lancet Neurol, 18(4), 357-375. doi:10.1016/S1474-4422(18)30454-X

2. French, J. A. (2007). Refractory epilepsy: clinical overview. Epilepsia, 48(Suppl 1), 3-7. doi:10.1111/j.1528-1167.2007.00992.x

3. Towanabut, S., Rungreangyingyod, L., \& Suthisisang, C. (1998). The prevalence and risk factors of refractory partial seizure Thai patients at Prasat Neurological Institute. J Med Assoc Thai, 81(7), 512-519

4. Sillanpaa, M., \& Shinnar, S. (2010). Long-term mortality in childhood-onset epilepsy. N Eng/ J Med, 363(26), 2522-2529. doi:10.1056/NEJMoa0911610

5. Kanchanatawan, B., \& Kasalak, R. (2012). Quality of life in Thai intractable epileptic patients with and without surgery. J Med Assoc Thai, 95(9), 1232-1238

6. Ramey, W. L., Martirosyan, N. L., Lieu, C. M., Hasham, H. A., Lemole, G. M. Jr., \& Weinand, M. E. (2013). Current management and surgical outcomes of medically intractable epilepsy. Clin Neurol Neurosurg, 115(12), 2411-2418. doi:10.1016/j.clineuro.2013.09.035

7. Shah, A. K., \& Mittal, S. (2014). Invasive electroencephalography monitoring: Indications and presurgical planning. Ann Indian Acad Neurol, 17(Suppl 1), S89-94. doi:10.4103/0972-2327.128668

8. Kumar, A., \& Chugani, H. T. (2013). The role of radionuclide imaging in epilepsy, Part 1: Sporadic temporal and extratemporal lobe epilepsy. J Nucl Med, 54(10), 1775-1781. doi:10.2967/jnumed.112.114397

9. Weil, S., Noachtar, S., Arnold, S., Yousry, T. A., Winkler, P. A., \& Tatsch, K. (2001). Ictal ECD-SPECT differentiates between temporal and extratemporal epilepsy: confirmation by excellent postoperative seizure control. Nucl Med Commun, 22(2), 233-237. doi:10.1097/00006231-200102000-00016

10. Chen, T., \& Guo, L. (2016). The role of SISCOM in preoperative evaluation for patients with epilepsy surgery: A meta-analysis. Seizure, 41, 43-50. doi:10.1016/j.seizure.2016.06.024

11. Leveille, J., Demonceau, G., De Roo, M., Rigo, P., Taillefer, R., Morgan, R. A., \& Walovitch, R. C. (1989). Characterization of technetium99m-L,L-ECD for brain perfusion imaging, Part 2: Biodistribution and brain imaging in humans. J Nucl Med, 30(11), 1902-1910 
12. Rabinowicz, A. L., Salas, E., Beserra, F., Leiguarda, R. C., \& Vazquez, S. E. (1997). Changes in regional cerebral blood flow beyond the temporal lobe in unilateral temporal lobe epilepsy. Epilepsia, 38(9), 1011-1014. doi:10.1111/j.1528-1157.1997.tb01484.x

13. Vallabhajosula, S., Zimmerman, R. E., Picard, M., Stritzke, P., Mena, I., Hellman, R. S., \& Goldsmith, S. J. (1989). Technetium-99m ECD: a new brain imaging agent: in vivo kinetics and biodistribution studies in normal human subjects. J Nucl Med, 30(5), 599-604

14. Ishizu, K., Yonekura, Y., Magata, Y., Okazawa, H., Fukuyama, H., Tanaka, F., \& Konishi, J. (1996). Extraction and retention of technetium99m-ECD in human brain: dynamic SPECT and oxygen-15-water PET studies. J Nucl Med, 37(10), 1600-1604

15. Jacquier-Sarlin, M. R., Polla, B. S., \& Slosman, D. O. (1996). Cellular basis of ECD brain retention. J Nucl Med, 37(10), 1694-1697

16. Ichise, M., Golan, H., Ballinger, J. R., Vines, D., Blackman, A., \& Moldofsky, H. (1997). Regional differences in technetium-99m-ECD clearance on brain SPECT in healthy subjects. J Nucl Med, 38(8), 1253-1260

17. Flores, L. G. 2, Jinnouchi, S., Nagamachi, S., Ohnishi, T., Futami, S., Nakahara, H., \& Tamura, S., nd, „,.. (1999). Retention of Tc-99m ECD in delayed SPECT of the brain. Ann Nucl Med, 13(1), 1-4. doi:10.1007/BF03165420

18. Grunwald, F., Menzel, C., Pavics, L., Bauer, J., Hufnagel, A., Reichmann, K., \& Biersack, H. J. (1994). Ictal and interictal brain SPECT imaging in epilepsy using technetium-99m-ECD. J Nucl Med, 35(12), 1896-1901

19. Tzourio-Mazoyer, N., Landeau, B., Papathanassiou, D., Crivello, F., Etard, O., Delcroix, N., \& Joliot, M. (2002). Automated anatomical labeling of activations in SPM using a macroscopic anatomical parcellation of the MNI MRI single-subject brain. Neuroimage, 15(1), 273-289. doi:10.1006/nimg.2001.0978

20. Engel, J. Jr (1993). Outcome with respect to epileptic seizures.Surgical Treatment of the Epilepsies,609-621.

\section{Figures}
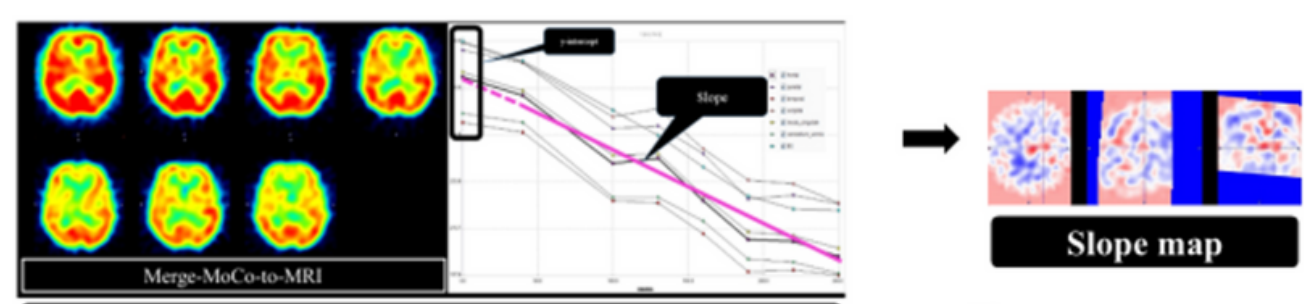

\section{Regression Model}

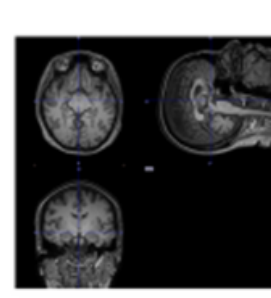

MRI

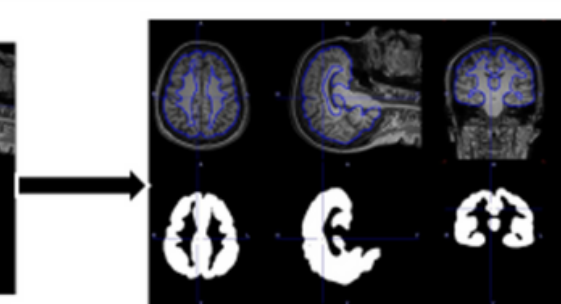

Grey-matter (GM) mask

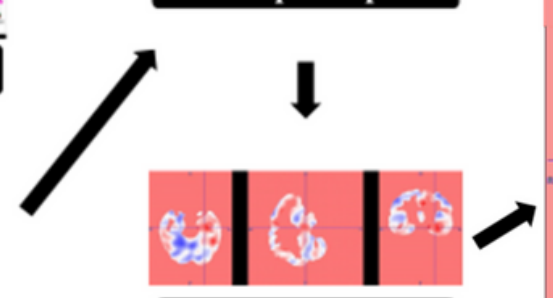

Slope map with GM mask

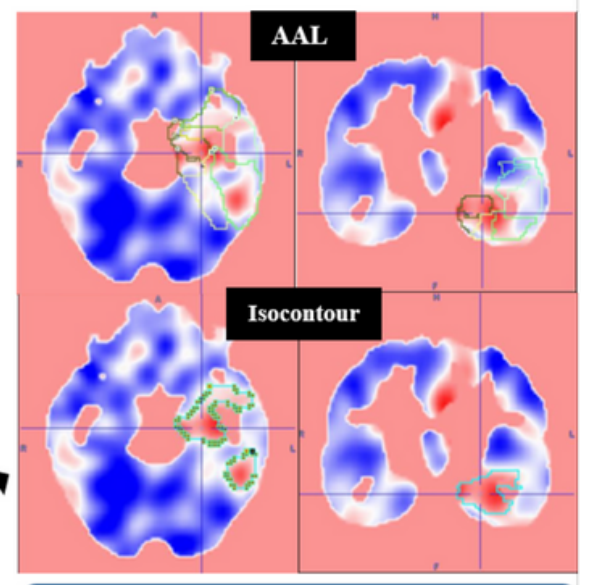

VOIs by iso-contouring using region growing inside AAL template

Figure 1

Image analysis process: 1) Merge seven serial images into time-sequence images 2) Motion correction and co-register to patient's T1weighted MRI images 3) Generate slope map (clearance rate from each image pixel) by pixelwise linear regression analysis 4) Create grey matter (GM) image using the patient's T1-weighted MRI to further mask the slope map 5) Draw regions of interest (ROIs) of suspected seizure onset zone by iso-contour (3D iso-contouring VOI tools using region-growing) inside the automated anatomical labeling (AAL) template atlas 


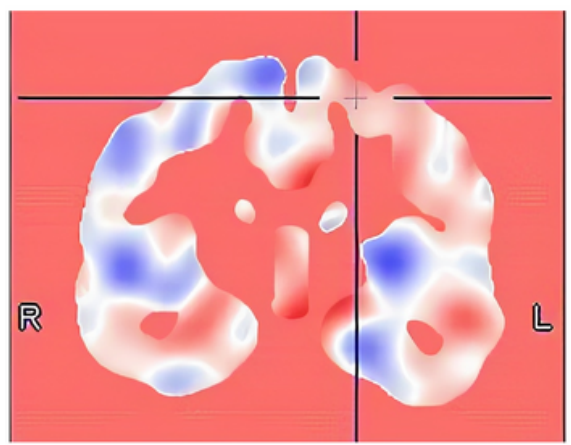

clearance rate $=0$

$\downarrow$

\section{fast washout \\ slow washout \\ washin}

\section{Figure 2}

The slope map pattern color bar and example: Example of slow washout pattern (crosshair cursor at the SOZ shows pale red color as compare with the pale blue color of the contralateral normal region (top). The slope map pattern displays in split BWR (blue-white-red) color bar (bottom). Background color represents clearance rate $=0.0$.
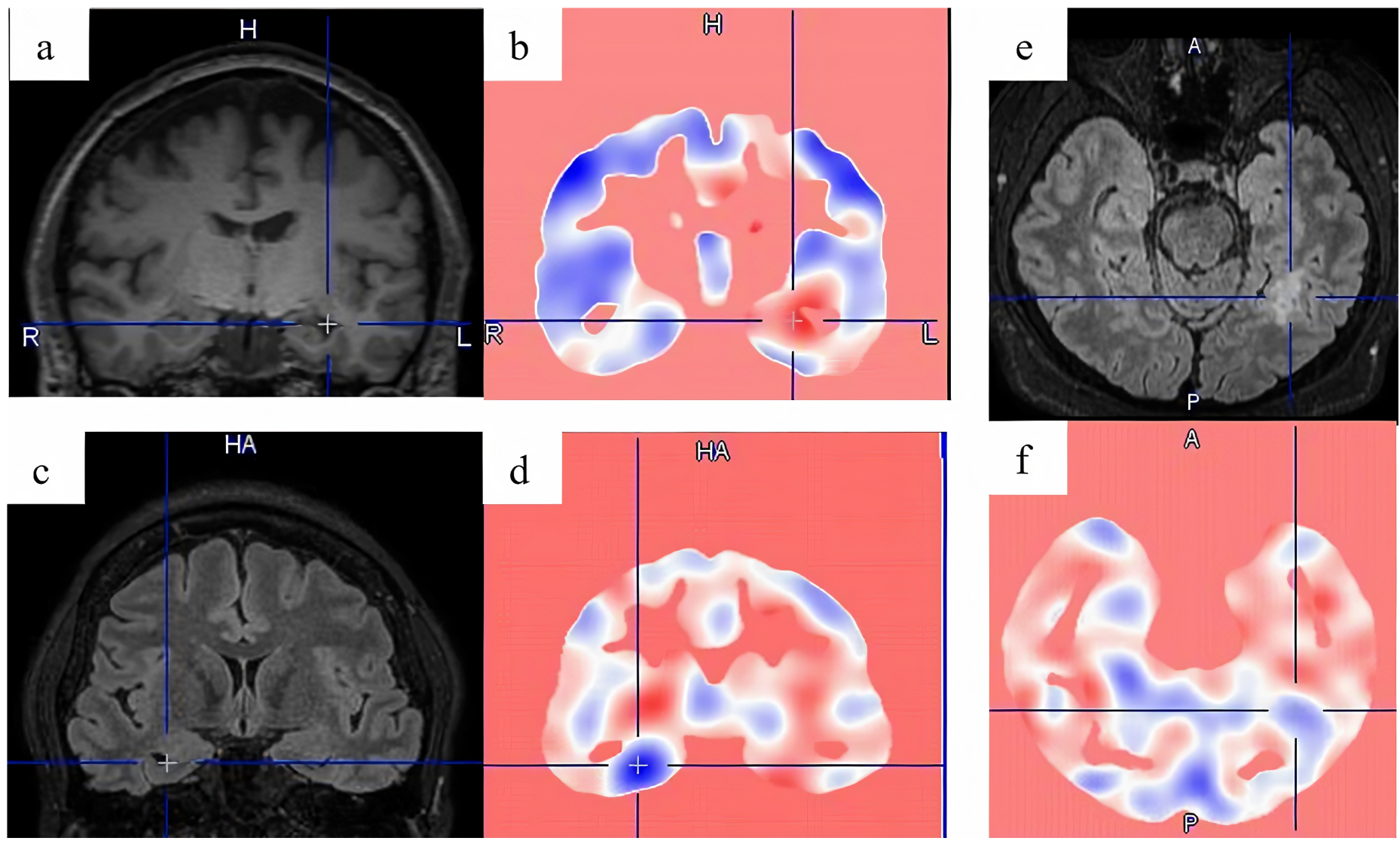

Figure 3 
Examples of slope maps in patients: Ictal slope map showed WASHIN (red) at left hippocampal sclerosis ( $a$, b crosshair cursor in patient number 2). Aura and interictal slope maps showed FAST WASHOUT (blue) at right hippocampal sclerosis (c, d crosshair cursor in patient number 3 ) and left fusiform lesion (e, f crosshair cursor in patient number 7), respectively. Background color represents clearance rate $=0.0$

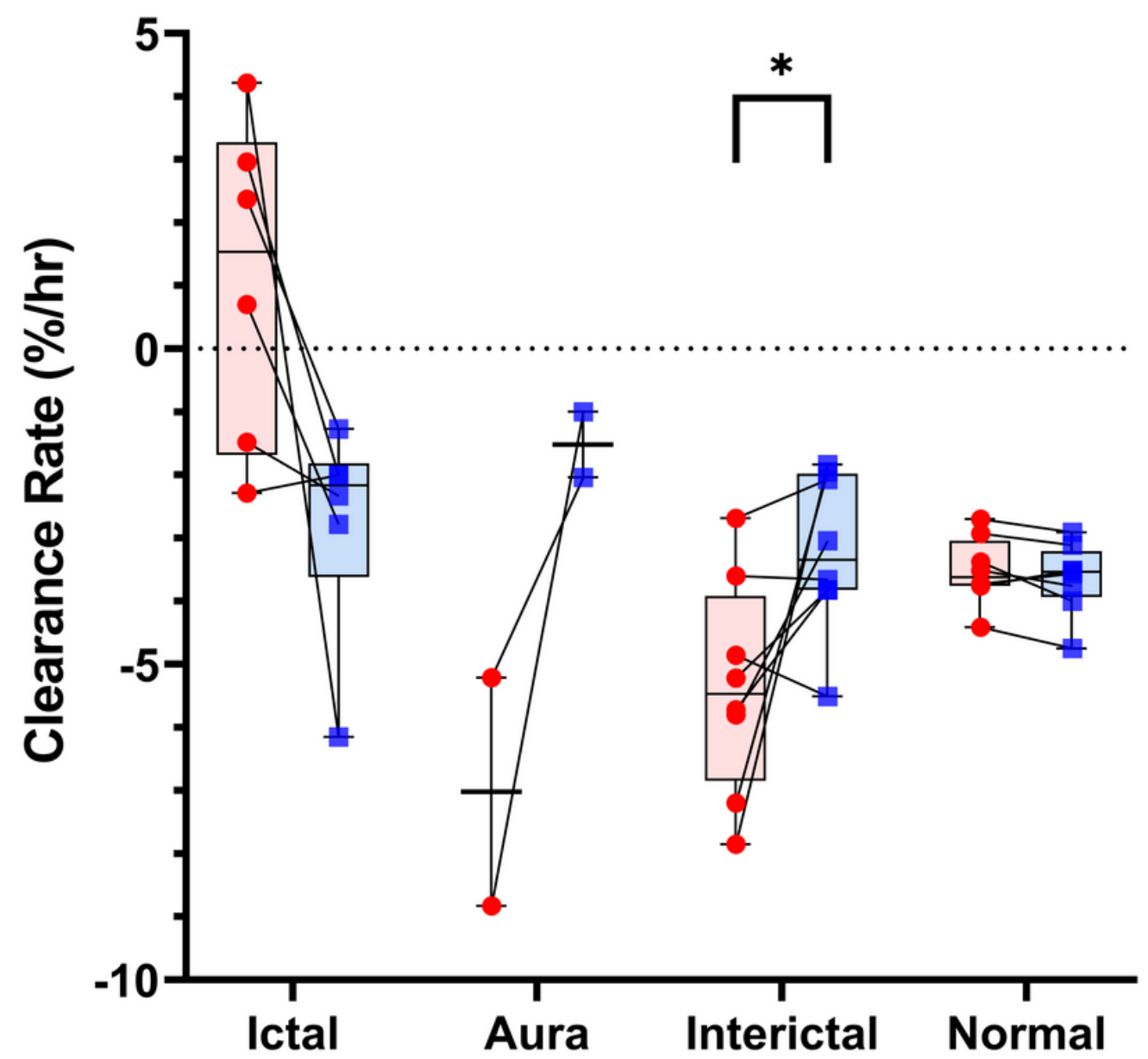

Figure 4

Comparison of ECD clearance rates (\%/hr) of seizure onset zones (SOZs) and the contralateral regions in three seizure states and normal: Paired t-tests between clearance rate of the $\mathrm{SOZ}$ and contralateral regions showed statistically significant difference in interictal state $(p=$ 0.039), but no statistically significant difference were seen in ictal state, aura state and normal

\section{Supplementary Files}

This is a list of supplementary files associated with this preprint. Click to download.

- BIBChecklist.docx

- Supplementarymaterial.pdf 